\section{TRANSITION TO ADULT CARE AUDIT CYCLE: IMPACT OF A CENTRAL TRANSITION TEAM}

Manorama Gadde, Manorama Gadde, Priya Narula. United Kingdom

10.1136/bmjpo-2021-RCPCH.42

Background Transitional healthcare is increasingly recognised as a priority for young people with chronic medical conditions. NICE quality standards for Transition were developed in 2016 to enable services to identify areas for improvement. ${ }^{1}$ We performed a trust-wide audit examining compliance with the NICE quality standards in 2017, implemented new measures, and repeated the audit in 2019. The new measures were led by a central transition team and included a transition section in electronic notes, electronic 'live' transition plan, learning from complaints and incidents, identifying transition champions, addressing organisation-wide barriers to transition, developing clear pathways for accessing support for patients with complex transitional needs, and obtaining feedback on transitional experience.

Objectives Objectives were

1. To assess compliance with NICE Transition Quality Standards

2. To assess whether measures implemented after the 2017 audit resulted in a change to compliance with NICE Transition Quality Standards

3. To review subgroups of patients, including complex patients requiring transition under three or more specialties, to assess whether more input is required in these particular patient groups.

Methods We reviewed two weeks of inpatient data (30th Sept - 13th Oct 2019) to identify patients with long-term medical conditions requiring transition, and assessed their transitional care against the NICE quality standards. The methodology was identical in 2017 and 2019.

1. Did discussions around transition begin by age 14 (or at diagnosis, if later)?

2. Was there an annual meeting where transition was discussed?

3. Was there evidence of a named transition worker?

4. Did they meet a practitioner from adult services before transferring? [partially assessed as most patients had not yet had their care transferred]

Results We identified 43 transition episodes in 2017 and 90 in 2019. Overall, transitional discussions had started in 70\% of patients, an improvement from 2017 (58\%). Most patients still had not started transition planning early (36\% 2017, $24 \%$ 2019) but more had an annual review (44\% 2017, 73\% $2019)$ and a named worker (20\% 2017, 48\% 2019). We identified three patients who had transferred to adult care without meeting an adult care provider. The transitional care of complex patients (under $3+$ specialties) had significantly improved across all criteria between the two audits with 100\% having started transition vs $66 \%$ in 2017.

Conclusions The 2019 audit demonstrated improvement overall, but identified areas requiring ongoing development particularly with early transitional planning. Next steps include agebased transition prompts in electronic notes, embedding links to transitional information in clinic letters, and arranging virtual visits to adult services.

\section{REFERENCES}

1. National Institute for Health and Care Excellence. (2016). Transition from children's to adults' services for young people using health or social care services (NICE Guideline NG43)

\section{SINGLE VERSUS SPLIT DOSE OF PREDNISOLONE IN THE TREATMENT OF RELAPSES OF CHILDHOOD NEPHROTIC SYNDROME}

Lasanthi Weerasooriya, Shenal Thalgahagoda, Asiri Abegunawardana. Sri Lanka

\subsection{6/bmjpo-2021-RCPCH.43}

\section{Background}

Introduction Childhood nephrotic syndrome follows a relapsing and remitting course. Corticosteroids are the mainstay of treatment and can be administered as a single early morning dose or as split doses. Usually, in the treatment of relapses, corticosteroids are administered daily until remission is achieved, followed by alternate day therapy. Early attainment of remission results in reduced morbidity and a reduced steroid burden.

Objectives To compare the time duration to attain remission using a single dose versus a split dose regime of prednisolone in the treatment of relapses of childhood nephrotic syndrome. Methods Children between 1 and 14 years of age admitted to a tertiary care paediatric nephrology unit from August 2019 to February 2020 with a relapse of nephrotic syndrome were randomised to two groups. Patients in Group A received oral prednisolone at $60 \mathrm{mg} / \mathrm{m}^{2}$ as a single morning dose while those in Group B received the same total dose as two divided doses( $2 / 3$ mane; $1 / 3$ vesper). The treatment regime was continued until remission was achieved following which all patients were switched to alternate day prednisolone at 60 $\mathrm{mg} / \mathrm{m}^{2}$ which was given as a single morning dose. The time duration from the commencement of prednisolone to the attainment of remission for the two groups was compared.

Results 104 children were included, of which 49(age 2.1613.8 years) received prednisolone as a split dose while 55(age 1.83-13.67 years) received a single morning dose. The mean duration to achieve remission was 5.04 days(SD 1.59; SE 0.22) for the split dose group and 6.74 days(SD 3.72;SE 0.50) for the single dose group. This difference was statistically highly significant. $[t(102)=2.967 ; p=0.001(<0.05)]$. There was no difference in the side effect profile.

Conclusions Use of prednisolone as a split dose results in earlier remission when compared to a single morning dose in the treatment of relapses of childhood nephrotic syndrome.

\section{GOING HOME SAFELY, A FAMILY CENTRED APPROACH: FACILITATING THE DISCHARGE PROCESS OF PRETERM INFANT FROM NEONATAL INTENSIVE CARE UNIT}

Mohammed Gaffari, Nuha Abdelghafar Nimeri, A Samawal. Hameed Mohd Lutfi, Mai Abdulla AL Qubaisi, Hilal Amin Tawfik Al Rifai, Nazla Abd El Monem Mohamed Mahmoud. Qatar

\subsection{6/bmjpo-2021-RCPCH.44}

Background Qatar is a sovereign state in the Middle East. Our Neonatal Intensive care unit in Woman's Wellness and Research Center(WWRC) is one of the largest Tertiary units in the Middle East with 20,000 deliveries per year and 4000 NICU admissions annually. Discharge process of preterm infants is a very complex issue that starts from the time of admission to discharge and follow-up post discharge. Preterm babies experience a much higher rate of hospital readmissions and death during the first year after birth compared with healthy term infants. Discharge plan should be individualized 